\title{
Оптически индуцированный зарядовый обмен в композитных структурах на основе ZnO с внедренными нанокристаллами $\mathrm{CsPbBr}_{3}$
}

\author{
() К.А. Дроздов ${ }^{1}$, И.В. Крылов ${ }^{1}$, А.С. Чижов ${ }^{2}$, М.Н. Румянцева ${ }^{2}$, Л.И. Рябова ${ }^{2}$, Д.Р. Хохлов ${ }^{1}$ \\ ${ }^{1}$ Московский государственный университет им. М.В. Ломоносова (физический фракультет), \\ 119991 Москва, Россия \\ ${ }^{2}$ Московский государственный университет им. М.В. Ломоносова (химический факультет), \\ 119991 Москва, Россия \\ E-mail: khokhlov@mig.phys.msu.ru
}

Поступила в Редакцию 10 декабря 2018 г.

В окончательной редакции 18 декабря 2018 г.

Принята к публикации 18 декабря 2018 г.

Внедрение в пористую матрицу $\mathrm{ZnO}$ нанокристаллов $\mathrm{CsPBr}_{3}$ приводит к появлению положительного отклика фотопроводимости в видимом спектральном диапазоне. Измерение спектральных зависимостей поглощения, фотолюминесценции и фотопроводимости показало, что форма и положение пика фотолюминесценции связаны с дефектами в $\mathrm{CsPbr}_{3}$. Анализ кинетики релаксации фотопроводимости показывает возможность ускорения процесса рекомбинации фотовозбужденных носителей заряда более чем на порядок. Обсуждаются механизмы, ответственные за эффект.

DOI: 10.21883/FTP.2019.06.47736.9042

\section{1. Введение}

Композитные структуры с галогенидными перовскитами являются перспективными материалами для различных направлений оптоэлектроники, в том числе для создания светоизлучающих диодов [1], фотоприемников и лазеров [2], элементов солнечных батарей [3]. Неорганические галогенидные перовскиты характеризуются высоким квантовым выходом фотолюминесценции [4], отличной стабильностью [5] и низкими токами пороговой генерации [6]. В ряде работ рассматривается возможность существенной модификации спектральных характеристик композотов с нанокристаллами $\mathrm{CsPbr}_{3}$ за счет эффекта размерного квантования [7], что позволяет получить узкий пик поглощения или фотолюминесценции в спектральном диапазоне 410-530 нм.

Ключевым элементом при создании диодов и солнечных элементов является композит на основе широкозонной пористой матрицы (например, ZnO) с внедренными в нее нанокристаллами $\mathrm{CsPbBr}_{3}$ [7]. Однако, поскольку нанокристалл $\mathrm{CsPbr}_{3}$ является трехкомпонентным, в нем неизбежно возникают дефекты. Дефекты могут влиять не только на свойства самого нанокристалла, но и на зарядовый обмен с другими частями композита.

Цель настоявшей работы - исследование процессов генерации и рекомбинации фотовозбужденных носителей заряда в композите на основе $\mathrm{ZnO}$ с внедренными нанокристаллами $\mathrm{CsPbr}_{3}$ при засветке в видимом спектральном диапазоне.

\section{2. Методика эксперимента и исследованные образцы}

Синтез нанокристаллического оксида цинка проведен методом осаждения из водного раствора $\mathrm{Zn}\left(\mathrm{CH}_{3} \mathrm{COO}\right)_{2}$ с использованием водного раствора $\mathrm{NH}_{4} \mathrm{HCO}_{3}$ при $50^{\circ} \mathrm{C}$ [8]. Осадок высушивался при $50^{\circ} \mathrm{C}$ в течение 24 ч, после чего проводился отжиг на воздухе при $300^{\circ} \mathrm{C}$ в течение 24 ч. Фазовый состав и параметры микроструктуры $\mathrm{ZnO}$ были определены методами рентгеновской дифракции и просвечивающей электронной микроскопии. Установлено, что полученный оксид цинка имеет структуру вюрцита, образован частицами размером 14-15 нм, объединенными в агломераты (рис. 1, $a$ ).

Синтез коллоидных нанокристаллов $\mathrm{CsPBr}_{3}$ проводился высокотемпературным инжекционным способом [9]. Полученные частицы имеют кубическую форму. Средний размер нанокристаллов определен из данных просвечивающей электронной микроскопии (рис. $1, b)$ и составил 10.2 нм.

Формирование нанокомпозитов для проведения измерений фотопроводимости осуществлялось непосредственно на микроэлектронном чипе $[10,11]$. Порошок нанокристаллического $\mathrm{ZnO}$ смешивался с органическим связующим ( $\alpha$-терпинеол), и небольшое количество полученной пасты переносилось на диэлектрическую подложку размерами $1.5 \times 1.5 \mathrm{MM}$, снабженную платиновыми измерительными контактами, с последующими сушкой при $70^{\circ} \mathrm{C}$ и отжигом при $350^{\circ} \mathrm{C}$ в течение суток. На поверхность сформированной пленки $\mathrm{ZnO}$ наносили 4 мкл золя нанокристаллов $\mathrm{CsPbr}_{3}$ в гексане (концентрация нанокристаллов 8-10 мг/мл), после чего полученную нанокомпозитную пленку подвергали сушке при $70^{\circ} \mathrm{C}$ на воздухе в течение 6 ч.

Поскольку используемые матрицы $\mathrm{ZnO}$ являются газовыми сенсорами резистивного типа [11], оптические и электрофизические свойства всей системы в значительной мере зависят от состояния поверхности пленки и, следовательно, от состава окружающей газовой среды. Все представленные в настоящей работе эксперимен- 

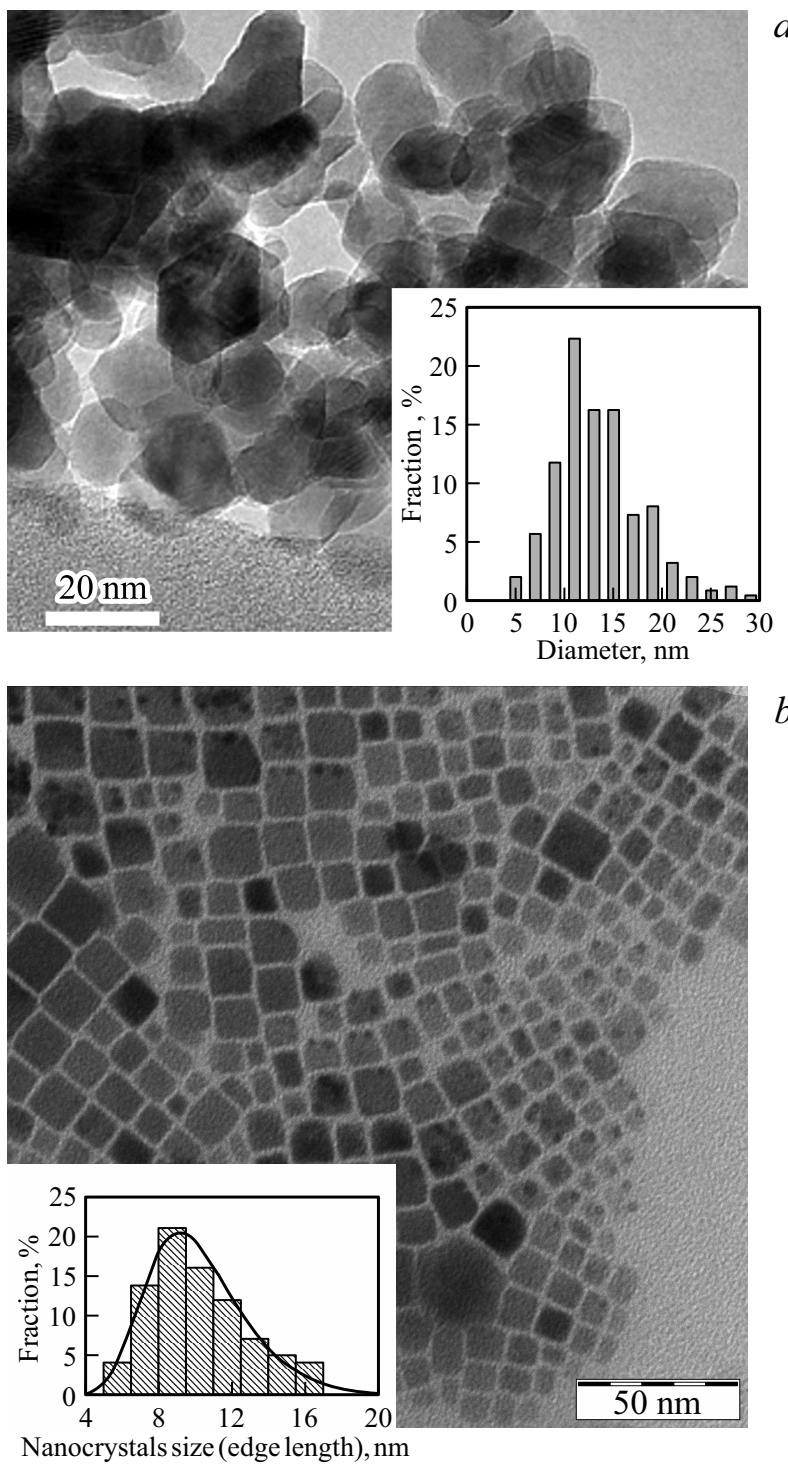

Рис. 1. Данные просвечивающей электронной микроскопии для кристаллитов матрицы $\mathrm{ZnO}(a)$ и нанокриталлов $\mathrm{CsPbBr}_{3}(b)$. На вставках — распределения соответствующих частиц по размерам.

тальные результаты были получены по единообразной методике. Образец устанавливался в измерительную камеру со смотровыми окнами, прозрачными в видимом и ближнем ультрафиолетовом (УФ) спектральных диапазонах. После этого осуществлялась стимулированная десорбция атомов с поверхности пленки за счет термического разогрева образца до $300^{\circ} \mathrm{C}$ с помощью нагревательного элемента резистивного типа, смонтированного на обратной стороне подложки. Затем измерительная камера откачивалась (давление после откачки составляло $10^{-3}$ атм) и заполнялась парами гелия, инертного для поверхности пленки $\mathrm{ZnO}$.

Матрица $\mathrm{ZnO}$ и композиты на ее основе имели электронную проводимость. Кинетика и спектры фотопроводимости исследованы при комнатной температуре.
В качестве подсветки использовался монохроматический источник на основе монохроматора МДР-206 и лампы белого света с известным спектром. Засветка образцов осуществлялась на длинах волн, отвечающих собственному оптическому поглощению широкозонной матрицы $\mathrm{ZnO}$ (390 нм) и нанокристаллов $\mathrm{CsPbBr}_{3}$ (480 и 515 нм). Длину волны $\lambda=515$ нм выбрали исходя из первопринципных расчетов положения дополнительного энергетического уровня, связанного с дефектообразованием в $\mathrm{CsPbBr}_{3}$ [12]. Длина волны $\lambda=480$ нм отвечает энергии, превышающей расстояние между нижним электронным и верхним дырочным уровнями в $\mathrm{CsPbBr}_{3}$, но не достаточной для межзонного возбуждения в $\mathrm{ZnO}$.

\section{3. Результаты измерений}

На рис. 2 представлены спектральные зависимости оптического поглощения и фотолюминесценции для золей $\mathrm{CsPbBr}_{3}$ в гексане. Пик фотолюминесценции сдвинут по отношению к краю поглощения в сторону бо́льших длин волн на 15 нм. Сопоставление длины волны, отвечающей краю поглощения в $\mathrm{CsPbBr}_{3}$, с известными из литературы данными [13] дает хорошее соответствие для линейных размеров нанокристаллов 10.2 нм, полученных из данных просвечивающей электронной микроскопии (рис. 1, b).

Типичные зависимости кинетики относительного сигнала фотопроводимости $\delta \sigma / \sigma_{0}\left(\sigma_{0}-\right.$ проводимость в темновых условиях, $\delta \sigma-$ изменение проводимости при подсветке) для структур с нанокристаллами показаны на рис. 3. Величина фотоотклика и время $(t)$ выхода на насыщение зависят от длины волны излучения накачки.

Поскольку релаксационные процессы имеют задержанный характер, для регистрации спектров фотопроводимости использовалась определенная методика [11]. Образец засвечивался в течение 20 с, а временной интервал между двумя последовательными измерениями составлял 30 мин. Спектральные зависимости снимались в несколько приемов, интервал между экспериментами составлял не менее 48 ч.

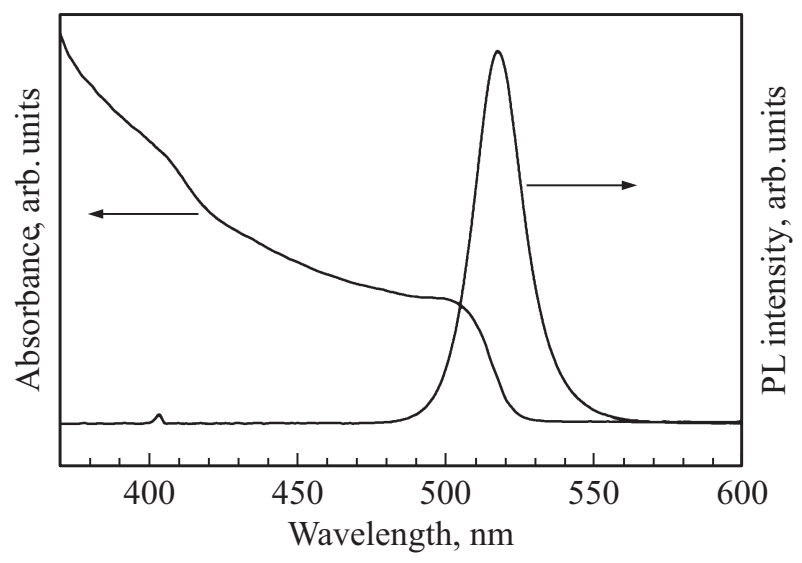

Рис. 2. Данные спектроскопии поглощения и фотолюминесценции (PL) для золей $\mathrm{CsPBr}_{3}$ в гексане. 


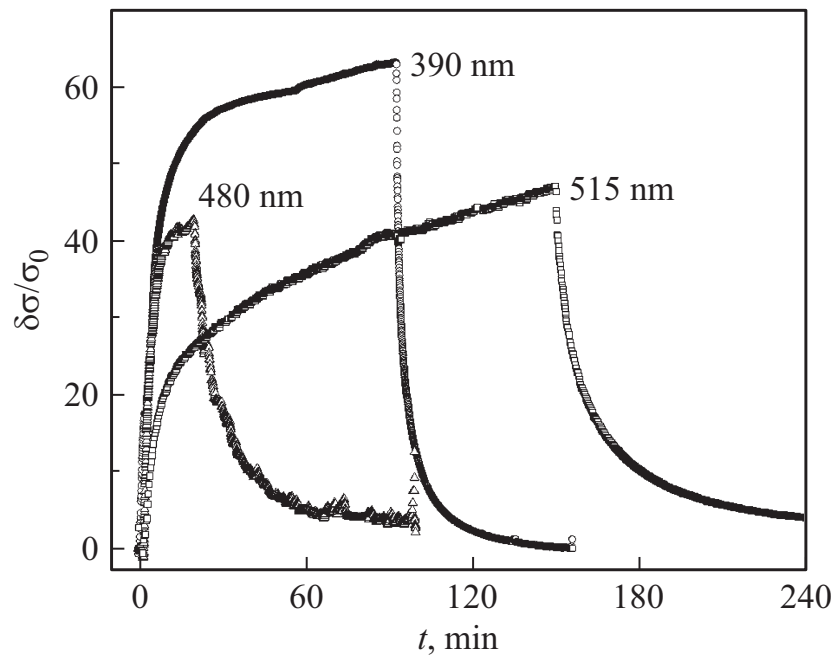

Рис. 3. Кинетики фотопроводимости $\delta \sigma / \sigma_{0}$ структур $\mathrm{ZnO}-\mathrm{CsPbBr}_{3}$. Длины волн излучения указаны.

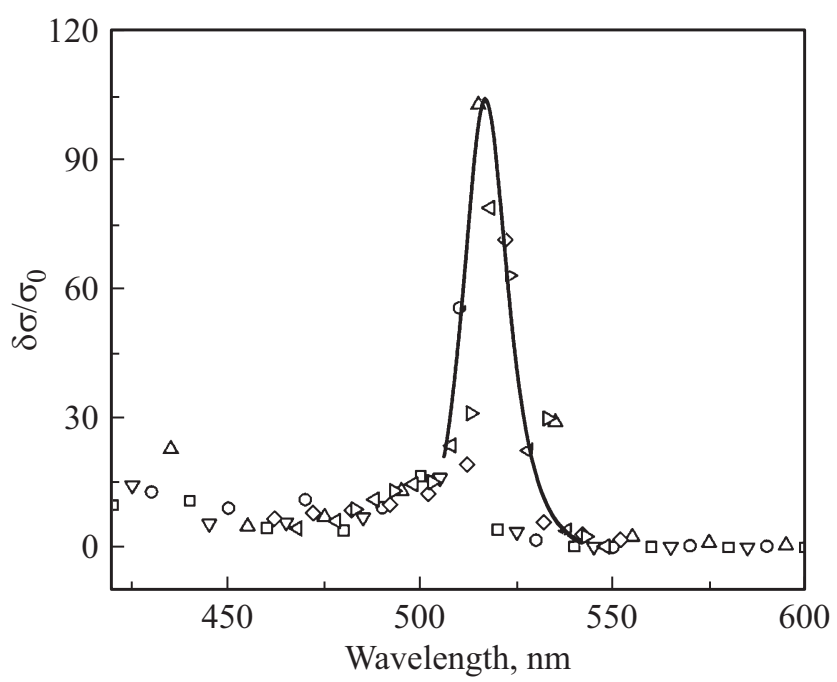

Pис. 4. Спектры фотопроводимости для структур $\mathrm{ZnO}-\mathrm{CdSe}$. Разные символы отвечают различным дням проведения эксперимента.

На рис. 4 представлены спектральные зависимости фотопроводимости для композитной структуры $\mathrm{ZnO}-\mathrm{CsPbr}_{3}$. Экспериментальные кривые содержат особенности, отвечающие оптическому поглощению нанокристаллов $\mathrm{CsPbBr}_{3}$ в гексане (рис. 2), а также присутствует дополнительный пик с существенно большей амплитудой и максимумом на 515 нм (выделен сплошной линией на рис. 4).

Важно, что все экспериментальные данные, представленные на рис. 4, хорошо воспроизводятся при проведении серии измерений, разнесенных по времени.

\section{4. Обсуждение результатов}

Спектральные зависимости фотопроводимости для композита $\mathrm{ZnO}-\mathrm{CsPbBr}_{3}$ обусловливаются оптическим поглощением матрицы $\mathrm{ZnO}$, нанокристаллов $\mathrm{CsPbBr}_{3}$ и механизмами переноса носителей заряда между составляющими композита. Матрица $\mathrm{ZnO}$ не фоточувствительна на длинах волн $\lambda>400$ нм. Положительный отклик в спектрах фотопроводимости (рис. 4) на данных длинах волн следует соотнести с вкладом от нанокристаллов $\mathrm{CsPbr}_{3}$. Наблюдаемый спектр фотопроводимости имеет сложную структуру и состоит из двух компонент. Кривая фотопроводимости в диапазоне 350-500 нм характеризуется относительно небольшой амплитудой фотоотклика и может быть однозначно соотнесена с собственным оптическим поглощением в $\mathrm{CsPbBr}_{3}$, связанным с уровнями размерного квантования (рис. 2). Пик с максимумом на 515 нм имеет существенно бо́льшую амплитуду (отношение величин проводимости на 515 и 500 нм составляет $\sim 5)$. При этом необходимо отметить, что положение пика достаточно хорошо отвечает наличию в энергетической диаграмме для $\mathrm{CsPbr}_{3}$ дополнительного энергетического уровня, связанного с дефектообразованием в трехкомпонентном нанокристалле [12]. В пользу данного утверждения также говорит достаточно малая ширина данного пика, поскольку основной вклад в уширение спектральных характеристик для системы коллоидных нанокристаллов при комнатной температуре вносит дисперсия по размеру. Отсутствие оптического поглощения на 515 нм для золей нанокристаллов $\mathrm{CsPbBr}_{3}$ можно объяснить принципиально различной методикой проведения измерений. Для измерений фотопроводимости использовалась импульсная засветка в течение 20 с, для поглощения - непрерывная засветка с существенно большей интенсивностью и длительное накопление сигнала. В последнем случае уровень дефектов мог оказаться полностью заполнен соответствующими носителями заряда и не вносить существенного вклада в процессы поглощения излучения от источника. Следует отметить, что спектральная зависимость фотолюминесценции для золей $\mathrm{CsPbBr}_{3}$ (рис. 2) крайне точно воспроизводит форму и положение рассмотренного выше пика фотопроводимости с максимумом на 515 нм, связанного с дефектным уровнем. Таким образом, фотолюминесценцию в $\mathrm{CsPbr}_{3}$ в условиях длительного возбуждения следует связать с излучательным рекомбинационным процессом с участием уровня дефектов.

Поскольку матрица $\mathrm{ZnO}$ не фоточувствительна на длинах волн $\lambda>400$ нм, кинетику фотопроводимости, полученную при подсветке композита $\mathrm{ZnO}-\mathrm{CsPbBr}_{3}$ на данных длинах волн (рис. 3), можно однозначно соотнести с вкладом от нанокристаллов $\mathrm{CsPbr}_{3}$. Положительная фотопроводимость обусловлена инжекцией неравновесных электронов из нанокристаллов в матрицу $\mathrm{ZnO}$. Подсветка структуры $\mathrm{ZnO}-\mathrm{CsPbBr}_{3}$ с энергией фотонов, превышающей ширину запрещенной зоны $\mathrm{ZnO}(\lambda<400$ нм), обеспечивает генерацию электроннодырочных пар как в матрице $\mathrm{ZnO}$, так и в нанокристаллах $\mathrm{CsPbr}_{3}$. Сопоставляя амплитуду фотопроводимости при засветке на разных длинах волн (рис. 3) и спектральные зависимости поглощения нанокристаллов $\mathrm{CsPbr}_{3}$ 


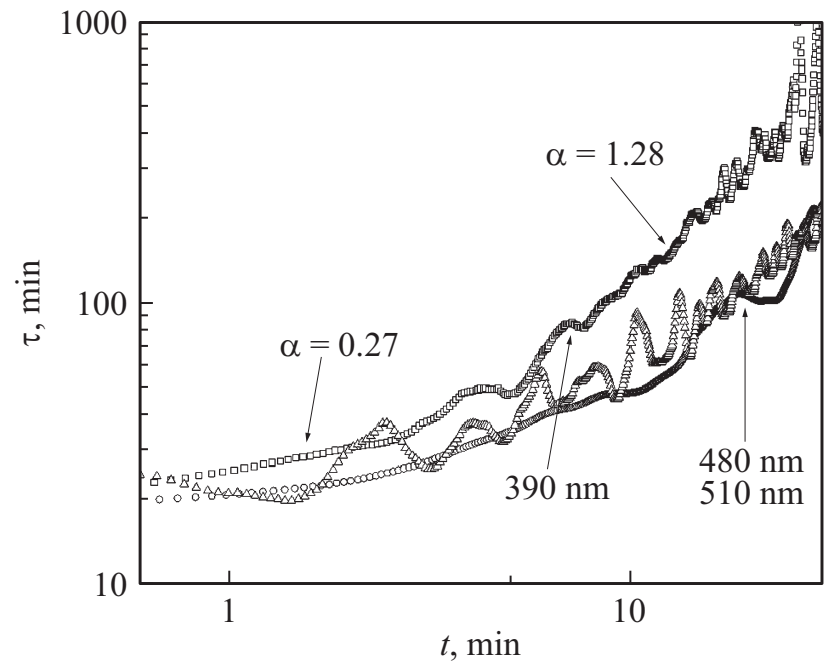

Рис. 5. Кинетики изменения мгновенного времени жизни $\tau$, характеризующего спад фотопроводимости после выключения подсветки. Длина волны излучения и значения коэффициента $\alpha$ в $\tau \propto t^{\alpha}$ указаны.

(рис. 2), можно утверждать что при подсветке с длиной волны $\lambda=390$ нм основной вклад в фотопроводимость обусловлен генерацией фотовозбужденных носителей заряда в самой матрице $\mathrm{ZnO}$. Таким образом, вся используемая подсветка (с длинами волн $\lambda=390,480,515$ нм) приводит в увеличению концентрации неравновесных электронов в матрице $\mathrm{ZnO}$. Транспорт носителей в пористых пленках $\mathrm{ZnO}$ определяется модуляцией зонного рельефа неоднородной полупроводниковой матрицы и наличием в энергетическом спектре рекомбинационного и дрейфового барьеров [14]. Установление квазиравновесия в системе при включении подсветки является долговременным процессом. Задержанный характер спада фотопроводимости определяется рекомбинационным барьером и зависит от механизмов рекомбинации.

Для анализа процессов релаксации фотовозбужденного неравновесного состояния использовались мгновенные времена релаксации $\tau=\sigma_{s} /|d \sigma / d t|\left(\sigma_{s}-\right.$ проводимость в стационарном состоянии) (рис. 5). Неэкспоненциальный спад проводимости может быть описан зависимостью $\tau(t) \propto t^{\alpha}$, где $\alpha$ характеризует механизмы рекомбинации [15]. Как видно из рис. 5, все кривые $\tau(t)$ имеют схожий вид. При этом для кривых можно выделить два характерных временны́х интервала, каждый с постоянным значением $\alpha: \alpha=0.27$ и 1.28 для временны́х интервалов $0-8$ и 10-60 мин соответственно. Поскольку для аналогичных матриц широкозонных оксидов без нанокристаллов характерные значения $\alpha$ близки к 1, можно утверждать, что релаксация фотовозбужденного состояния в $\mathrm{ZnO}-\mathrm{CsPbBr}_{3}$ идет с учетом процессов в нанокристаллах $\mathrm{CsPbBr}_{3}$.

Для временно́го интервала $0-8$ мин $\alpha=0.27$, что в целом отвечает значениям для релаксации за счет туннелирования электронов из матрицы в нанокристаллы с последующей рекомбинацией [16]. При изначальной засветке на длинах волн 480, 515 нм фотовозбужденые носители рождаются в нанокристаллах $\mathrm{CsPbr}_{3}$ с последующей инжекцией электронов в матрицу $\mathrm{ZnO}$ и локализацией дырок в $\mathrm{CsPBr}_{3}$. Рекомбинация происходит при туннелировании электронов из матрицы на уровни размерного квантования в $\mathrm{CsPbBr}_{3}$. При изначальной засветке на длинах волн 390 нм фотовозбужденные носители рождаются в матрице $\mathrm{ZnO}$. При этом дырки могут туннелировать в нанокристаллы с последующей локализацией в них. Рекомбинация происходит аналогично процессу, изложенному выше. Различный механизм пространственного разделения фотовозбужденных носителей (туннелирование с уровней размерного квантования $\mathrm{CsPbr}_{3}$ в зону проводимости $\mathrm{ZnO}$ для генерации в нанокристаллах и из валентной зоны $\mathrm{ZnO}$ на уровни размерного квантования $\mathrm{CsPbr}_{3}$ для генерации в $\mathrm{ZnO}$ ) обусловливает различные времена выхода фотопроводимости структур на насыщение (рис. 3). Туннельные процессы обеспечивают длительные времена выхода на насыщение при подсветке и задержанную фотопроводимость.

Для временно́го интервала 10-60 мин $\alpha=1.28$, что не характерно ни для чистых матриц, ни для композитных материалов на основе $\mathrm{ZnO}$ с внедренными нанокристаллами другого состава $[10,11,16]$. Существенное отличие композита $\mathrm{ZnO}-\mathrm{CsPbBr}_{3}$ заключается в наличии дополнительного уровня в $\mathrm{CsPbr}_{3}$, связанного с дефектами и лежащего ниже нижнего электронного уровня размерного квантования. Отсюда наиболее вероятным представляется следующий процесс. При выключении подсветки происходит уменьшение концентрации фотовозбужденных электронов в матрице $\mathrm{ZnO}$ (начальный временной интервал $0-8$ мин с $\alpha=0.27)$, что приводит к смещению положения квазиуровня Ферми в $\mathrm{ZnO}$ вниз по энергии. Когда квазиуровень Ферми приближается к значению энергии, отвечающему уровню дефектов в $\mathrm{CsPbBr}_{3}$, активируется дополнительный механизм переноса электронов из матрицы на этот уровень. Совокупность двух туннельных процессов переноса электронов из матрицы $\mathrm{ZnO}$ в нанокристаллы позволяет достичь существенно более быстрой рекомбинации. Релаксация к равновесному значению осуществляется за время $\sim 1$ ч, в то время как для аналогичных композитов с нанокристаллами CdSe релаксация занимает > 24 ч [16].

\section{5. Заключение}

Исследованы спектральные зависимости поглощения квантовых точек $\mathrm{CsPbr}_{3}$, спектры фотопроводимости и кинетика задержанной фотопроводимости композита $\mathrm{ZnO}-\mathrm{CsPbBr}_{3}$ при дополнительной подсветке на длинах волн 390, 480, 551 нм.

Под действием внешнего излучения концентрация носителей заряда в матрице $\mathrm{ZnO}$ может изменяться за счет межзонной генерации в $\mathrm{ZnO}$ и инжекции из нанокристаллов $\mathrm{CsPbBr}_{3}$. При межзонной генерации в $\mathrm{ZnO}$ фотовозбужденные электроны остаются в матрице, дырки туннелируют в нанокристаллы $\mathrm{CsPbr}_{3}$. 
При генерации носителей заряда в $\mathrm{CsPbBr}_{3}$ электроны туннелируют в матрицу $\mathrm{ZnO}$, дырки остаются локализованными в нанокристаллах $\mathrm{CsPbr}_{3}$. Независимо от типа фотогенерации неравновесных носителей заряда рекомбинация происходит в нанокристаллах $\mathrm{CspBr}_{3}$. Излучательная рекомбинация в $\mathrm{CsPbr}_{3}$ идет с участием уровня дефектов, лежащего ниже нижнего электронного уровня размерного квантования. Вследствие этого пик фотолюминесценции существенно менее зависит от дисперсии нанокристаллов по размеру - полуширина пика составляет $\sim 20$ нм.

В зависимости от взаимного расположения квазиуровня Ферми в $\mathrm{ZnO}$ и уровня дефектов в $\mathrm{CsPbBr}_{3}$ процесс рекомбинации может претерпевать изменения. В случае, когда квазиуровень Ферми лежит ниже или на одной высоте с уровнем дефектов, активируется дополнительный процесс переноса носителей заряда из матрицы $\mathrm{ZnO}$ в нанокристаллы $\mathrm{CsPbBr}_{3}$. Данный механизм позволяет сократить характерные времена релаксации фотопроводимости структуры более чем на порядок, что существенно повышает привлекательность композитных структур с $\mathrm{CsPbBr}_{3}$ для практического применения.

Таким образом, показано, что характер задержанной фотопроводимости в композитных структурах со специально внедренными неонородностями нанометрового масштаба в значительной мере определяется расположением квазиуровня Ферми в матрице. Данное обстоятельство существенно отличает рассматриваемую ситуацию от фотопроводимости в легированных полупроводниках на основе $\mathrm{Pb}_{1-x} \mathrm{Sn}_{x} \mathrm{Te}(\mathrm{In})$, в которых характер задержанной фотопроводимости практически не изменяется в зависимости от расположения квазиуровня Ферми в энергетическом спектре полупроводника [17]

Работа выполнена при поддержке гранта РНФ № 1612-10071. Синтез нанокристаллов $\mathrm{CsPbBr}_{3}$ выполнен при поддержке гранта РФФИ № 18-33-01004.

\section{Список литературы}

[1] Z.K. Tan, R.S. Moghaddam, M.L. Lai, P. Docampo, R. Higler, F. Deschler, M. Price, A. Sadhanala, L.M. Pazos, D. Credgington, F. Hanusch, T. Bein, H.J. Snaith, R.H. Friend. Nature Nanotechnol., 9, 687 (2014).

[2] L. Su, Z.X. Zhao, H.Y. Li, J. Yuan, Z.L. Wang, G.Z. Cao, G. Zhu. ACS Nano, 9, 11310 (2015).

[3] N.J. Jeon, J.H. Noh, Y.C. Kim, W.S. Yang, S. Ryu, S.I. Seok. Nature Materials, 13, 897 (2014).

[4] M. Kulbak, S. Gupta, N. Kedem, I. Levine, T. Bendikov, G. Hodes, D. Cahen. J. Phys. Chem. Lett., 7 (1), 167 (2016).

[5] S. Huang, Z. Li, L. Kong, N. Zhu, A. Shan, L. Li. J. Am. Chem. Soc., 138, 5749 (2016).

[6] Y. Wang, X. Li, X. Zhao, L. Xiao, H. Zeng, H. Sun. Nano Lett., 16, 448 (2016).

[7] Xiafang Du, Guan Wu, Jian Cheng, Hui Dang, Kangzhe Ma, Ya-Wen Zhang, Peng-Feng Tan, Su Chen. RSC Adv., 7, 10391 (2017).

[8] N. Vorobyeva, M. Rumyantseva, D. Filatova, E. Konstantinova, D. Grishina, A. Abakumov, S. Turner, A. Gaskov. Sensors Actuators B: Chemical, 182, 555 (2013).
[9] L. Protesescu, S. Yakunin, M.I. Bodnarchuk, F. Krieg, R. Caputo, C.H. Hendon, R.X. Yang, A. Walsh, M.V. Kovalenko. Nano Lett., 15 (6), 3692 (2015).

[10] A.S. Chizhov, M.N. Rumyantseva, R.B. Vasiliev, D.G. Filatova, K.A. Drozdov, I.V. Krylov, A.M. Abakumov, A.M. Gaskov. Sensors Actuators B: Chemical, 205, 305 (2014).

[11] A. Chizhov, M. Rumyantseva, R. Vasiliev, D. Filatova, K. Drozdov, I. Krylov, A. Marchevsky, O. Karakulina, A. Abakumov, A. Gaskov. Thin Sol. Films, 618, 253 (2016).

[12] Mingzhi Zhang, Zhiping Zheng, Qiuyun Fu, Pengju Guo, Sen Zhang, Cheng Chen, Hualin Chen, Mei Wang, Wei Luo, Yahui Tian. J. Phys. Chem. C, 122 (19), 10309 (2018).

[13] L. Protesescu, S. Yakunin, M.I. Bodnarchuk, F. Krieg, R. Caputo, C.H. Hendon, Ruo Xi Yang, A. Walsh, M.V. Kovalenko. Nano Lett., 15 (6), 3692 (2015).

[14] Р.Б. Васильев, С.Г. Дорофеев, М.Н. Румянцева, Л.И. Рябова, А.М. Гаськов. ФТП, 40 (1), 108 (2006).

[15] М.К. Шейнкман, А.Я. Шик. ФТП, 10, 209 (1976).

[16] К.А. Дроздов, И.В. Крылов, А.С. Чижов, М.Н. Румянцева, Л.И. Рябова, Д.Р. Хохлов. ФТП, 7, 763 (2018).

[17] Л.И. Рябова, Д.Р. Хохлов. УФН, 184, 1033 (2014).

Редактор Л.В. Шаронова

\section{Optically induced charge exchange in composite structures based on $\mathrm{ZnO}$ with embedded $\mathrm{CsPbBr}_{3}$ nanocrystals}

\author{
K.A. Drozdov ${ }^{1}$, I.V. Krylov' ${ }^{1}$, A.S. Chizhov ${ }^{2}$, \\ M.N. Rumyantseva ${ }^{2}$, L.I. Ryabova ${ }^{2}$, D.R. Khokhlov ${ }^{1}$ \\ ${ }^{1}$ Lomonosov Moscow State University \\ (Department of Physics), \\ 119991 Moscow, Russia \\ 2 Lomonosov Moscow State University \\ (Department of Chemistry), \\ 119991 Moscow, Russia
}

Abstract The introduction of $\mathrm{CsPbBr}_{3}$ nanocrystals into the $\mathrm{ZnO}$ porous matrix leads to appearance of a positive photoconductivity response in the visible spectral range. Measurement of the spectral dependences of absorption, photoluminescence and photoconductivity showed that the shape and position of the photoluminescence peak are associated with defects in $\mathrm{CsPbBr}_{3}$. Analysis of the photoconductivity relaxation kinetics shows the possibility of accelerating the photoexcited charge carriers recombination process by more than an order of magnitude. The mechanisms responsible for the effect are discussed. 